DEAL WATCH

\section{Merck bets on purine receptor revival}

\begin{abstract}
Merck (known as MSD outside the United States and Canada) has announced it will acquire Afferent Pharmaceuticals in a deal worth up to US\$1.25 billion in upfront and milestone payments (FIG. 1). Afferent has developed antagonists for $\mathrm{P} 2 \mathrm{X}$ purinoceptor 3 (P2X3), a ligand-gated ion channel that is expressed on neurons and which could have applications in numerous neurogenic conditions, including chronic cough, neuropathic pain and refractory hypertension.

The purinoceptors of the P2 family are activated by ATP, a neuronal cotransmitter that is released by components of the nervous system as well as by dying cells. Family members such as $\mathrm{P} 2 \mathrm{X} 7$, which is activated only by high concentrations of ATP, are likely to have roles predominantly in apoptosis and inflammation, whereas members such as $\mathrm{P} 2 \mathrm{X} 3$, which is activated at low concentrations, are potential therapeutic targets for diseases caused by aberrant neural transmission.
\end{abstract}

The P2X family of purinoceptors attracted considerable attention in the early 2000s, but negative efficacy data in clinical trials, particularly those examining $\mathrm{P} 2 \mathrm{X} 7$ inhibitors for the treatment of rheumatoid arthritis, cooled the field substantially. Indeed, Roche terminated their $\mathrm{P} 2 \mathrm{X} 3$ programme around this time, and programme leader Anthony Ford left Roche and founded Afferent Pharmaceuticals, where he continued working on the compounds identified at Roche. Afferent's most advanced P2X3-targeting Roche high-throughput screening hit.

AF-219 is an allosteric P2X 3 antagonist. Targeting the allosteric site on P2X ATP-binding site, helps in identifying suitably drug-like compounds, as well as providing sufficient selectivity, given the multiple other receptors that share a similar ATP-binding site. Christa Müller, a professor at the University of Bonn, Germany, who has developed compounds targeting a related receptor, $\mathrm{P} 2 \mathrm{X} 4$, says that $\mathrm{AF}-219$ "seems to be a very good allosteric compound, with good pharmacokinetic and physicochemical properties".

Müller thinks that $\mathrm{P} 2 \mathrm{X} 3$ and $\mathrm{P} 2 \mathrm{X} 4$ are good targets for neuropathic pain and other neurogenic conditions, but that the multiple, often contrasting roles that $\mathrm{P} 2 \mathrm{X} 7$ has in compound, AF-219, originated from a receptors, rather than the highly polar different pathologies could make it a difficult target and further studies will be required to find an appropriate indication and therapeutic window. She hopes the successes of Afferent will restore confidence in the therapeutic potential of targeting members of the P2X family. "I think this is very good news for the field," she says.

AF-219 recently showed clinical efficacy in a Phase llb trial of patients with chronic cough, and is currently in a Phase II trial in patients with idiopathic pulmonary fibrosis and cough (see Further information). "Cough is really a respiratory manifestation of pain - it's through activation of sensory nerves," explains Peter Barnes, head of respiratory medicine at the National Heart and Lung Institute in London, UK. Although codeine and stronger opiates can be used to treat chronic idiopathic cough, their addictive properties preclude their widespread use. "Chronic idiopathic cough has really been untreatable," says Barnes. He wonders whether these compounds might also be useful to treat post-viral cough, which is underscored by an increased neural sensitivity that can go on for weeks after viral clearance. "That's the commonest cough in the population and many people are taking cough medicines that they buy over the counter, which are almost completely ineffective," he says.

Afferent's second asset, AF-130, is also an allosteric $\mathrm{P} 2 \mathrm{X} 3$ inhibitor and is being investigated in a Phase I clinical trial for resistant hypertension. Both resistant hypertension and chronic cough are seemingly unusual first indications for compounds whose targets have known roles in neuropathic pain, but both of these conditions involve neuronal hypersensitivity.
Indeed, chronic idiopathic cough and resistant hypertension could be good diseases in which to test the efficacy of neuromodulatory compounds before potentially expanding the indication to include pain from other causes. First, cough and hypertension can be monitored through cough recorder devices and blood pressure monitors, respectively, which provides an opportunity to objectively measure the consequences of altered neural transmission in a clinical trial. Barnes points out that cough monitors allow you to "document a clear effect, whereas effects on pain are obviously more subjective". Second, there are no currently available treatments for chronic idiopathic cough, so there is substantial unmet medical need and the barrier to market entry should be relatively low. If compounds are effective in these conditions, they could subsequently be explored for the treatment of other conditions. "Chronic idiopathic cough does seem to be explained by increased neural hypersensitivity, so it's a model of something that is important in neuropathic pain," says Barnes.

Although the initial excitement about $\mathrm{P} 2 \mathrm{X} 7$ as a therapeutic target for rheumatoid arthritis has died down, $\mathrm{P} 2 \mathrm{X} 7$ could also be an interesting target for pain, including inflammatory or cancer-associated pain. P2X7 is also being investigated as a target in cancer, although the receptor would have to be activated in those circumstances. Numerous P2X family members could be important therapeutic targets. "I think P2X receptors really have a great future, because they're involved in pain and inflammation, which are related to many pathological conditions," notes Müller.

\section{Megan Cully}

FURTHER INFORMATION

Afferent's Phase llb trial press release:

http://www.afferentpharma.com/news/092815/

Clinical trials database: http://www.clinicaltrials.gov ALL LINKS ARE ACTIVE IN THE ONLINE PDF
Merck to acquire Afferent Pharmaceuticals

Deal type: acquisition

- Acquiring company: Merck (known as MSD

outside United States and Canada)

- Acquired company: Afferent Pharmaceuticals
Date announced: 9 June 2016

Value: up to US\$1.25 billion

- \$500 million upfront

- Up to $\$ 750$ million additional in milestone payments
Asset characteristics

- Afferent's discovery programme for P2X3 receptor inhibitors, which are implicated in neurological conditions such as neuropathic pain

- Afferent's most advanced compound, AF-219, has completed a Phase llb clinical trial for refractory chronic cough and is in a Phase II trial for idiopathic pulmonary fibrosis with cough

- Afferent's second asset, AF-130, is being investigated in a Phase I clinical trial for resistant hypertension

Figure 1 | Deal snapshot. 